\title{
Conhecimento sobre a farmacoterapia por portadores de Hipertensão Arterial Sistêmica
}

\author{
Knowledge about pharmacotherapy among hypertensive patients
}

Fabiane Raquel Motter ${ }^{1}$

Maria Teresa Anselmo Olinto ${ }^{1}$

Vera Maria Vieira Paniz ${ }^{1}$
${ }^{1}$ Programa de PósGraduação em Saúde Coletiva, Universidade do Vale do Rio dos Sinos. Av. Unisinos 950, Cristo Rei. 93022-000 São Leopoldo RS.wmotter@terra.com.br
Abstract The scope of this study was to evaluate the knowledge of pharmacotherapy among users of arterial hypertension medication. A quantitative cross-sectional study was conducted with adults aged 20 years or older, who were users of antihypertensive drugs dispensed by the Public Pharmacy of São Francisco de Paula in the State of Rio Grande do Sul. The study evaluated the knowledge of patients about the drug name, therapeutic indication, dose, administration routine, and duration of treatment, attributing a score (0-5). Multiple linear regression was used to investigate the relationship between the patients' knowledge and characteristics and 678 medication users were interviewed. The mean knowledge score was $3.27(S D=1.16)$. Lower age, higher schooling, Caucasian, better self-reported health and lower number of prescribed drugs were associated with higher knowledge scores. It was observed that the patients' knowledge of all the aspects evaluated is limited, a result that could compromise the correct and safe use of the drugs, and suggesting the need for better counseling and follow-up of users, to control this morbidity.

Key words Health knowledge, Health attitudes and practices, Hypertension, Pharmacotherapy, Antihypertensive drugs
Resumo O objetivo do estudo foi avaliar o conhecimento sobre a farmacoterapia em usuários de medicamentos para hipertensão arterial. Estudo quantitativo transversal com adultos de 20 anos ou mais de idade, usuários de anti-hipertensivos dispensados na Farmácia Básica de São Francisco de Paula, RS. Avaliou-se o conhecimento sobre o nome do medicamento, indicação terapêutica, dose, frequência/horários de administração e duração do tratamento, por meio de um escore (0-5). Utilizou-se regressão linear múltipla para investigar a relação com características dos usuários. Entrevistou-se 678 usuários de medicamentos. A média de escore de conhecimento foi de 3,27 ( $d p=1,16)$. Menor idade, maior escolaridade, cor da pele branca, melhor percepção de saúde e menor número de medicamentos prescritos mostraram-se associados a um maior escore de conhecimento. Observou-se um conhecimento limitado em relação a todos os aspectos avaliados, o que pode comprometer o uso correto e seguro da farmacoterapia, sugerindo a necessidade de melhor orientação e acompanhamento dos usuários para o controle dessa morbidade.

Palavras-chave Conhecimentos, atitudes e prática em saúde, Hipertensão, Farmacoterapia, Anti-hipertensivos 


\section{Introdução}

A Hipertensão Arterial Sistêmica (HAS) é considerada, atualmente, um grave problema de saúde pública, não apenas por apresentar alta prevalência, mas também por representar um dos principais fatores de risco para doenças cardiovasculares, cerebrovasculares e doença renal crônica. A HAS é responsável por $40 \%$ das mortes por acidente vascular cerebral e $25 \%$ das por síndrome coronariana aguda, sendo que esta porcentagem aumenta proporcionalmente aos valores pressóricos $^{1,2}$. Estudos nacionais revelam que a sua prevalência afeta de $22-44 \%$ da população adulta ${ }^{3-8}$.

A HAS é uma condição clinica multifatorial caracterizada por níveis elevados e sustentados de pressão arterial (PA). É definida pela persistência de pressão arterial sistólica acima de 135 $\mathrm{mmHg}$ e diastólica acima de $85 \mathrm{mmHg}$. O controle desta morbidade consiste tanto no tratamento não farmacológico, representados pela adoção de um estilo de vida saudável, quanto no tratamento farmacológico mediante o uso de medicamentos, ou ainda, na associação de am$\operatorname{bos}^{1,7}$. Entretanto, sabe-se que a utilização de medicamentos para o controle e a manutenção dos níveis tensionais em níveis considerados normais é indicada em mais de $70 \%$ dos casos ${ }^{6,9}$.

No Brasil, o Ministério da Saúde, instituiu o Plano Nacional de Reorganização da Atenção à Hipertensão Arterial e ao Diabetes Mellitus (DM), que visa o acompanhamento de forma sistemática e integral dos portadores de HAS e DM, além de garantir o acesso gratuito aos medicamentos essenciais para o seu controle. Neste contexto, a atenção básica é responsável por desenvolver ações voltadas à promoção da saúde, prevenção de agravos, tratamento e recuperação, exercendo assim, um papel fundamental no controle da $\mathrm{HAS}^{10}$.

Apesar desses esforços, estudos revelam que somente um terço dos portadores de HAS possui níveis tensionais controlados ${ }^{3,6}$. Diversos fatores podem ter influência sobre o controle da HAS, entre estes está o adequado conhecimento dos portadores sobre o tratamento farmacológico, considerado um fator importante para a utilização dos medicamentos conforme a prescrição médica ${ }^{11-13}$.

$\mathrm{Na}$ literatura revisada foram identificados vários estudos que avaliam o conhecimento sobre a farmacoterapia da HAS, entretanto, observa-se que os aspectos analisados são distintos. Alguns estudos avaliaram a duração do tratamento e/ou o nome do medicamento, por considerarem este último como um preditor do co- nhecimento ${ }^{14-27}$. Outros, avaliaram a dose e/ou a frequência/ horários de administração $0^{22,28-30}$, sendo realizados em populações específicas e com amostras pequenas. Outros, ainda, avaliaram a indicação terapêutica do medicamento ${ }^{14,22,25,28-32}$. Não foram encontrados estudos abordando todos os aspectos do conhecimento acima citados e somente um estudo avaliou os fatores associados ao conhecimento ${ }^{24}$.

Em vista disso, este estudo tem como objetivo avaliar o conhecimento sobre a farmacoterapia, o que inclui o nome do medicamento, indicação terapêutica, dose, frequência/horários de administração e duração do tratamento em usuários de medicamentos anti-hipertensivos e identificar os fatores associados a este conhecimento.

\section{Métodos}

Foi realizado um estudo transversal com adultos de 20 anos ou mais de idade, usuários da Farmácia Básica do município de São Francisco de Paula, RS, situado a $112 \mathrm{~km}$ da capital, Porto Alegre. O município possui aproximadamente 20 mil habitantes e uma estrutura de saúde que inclui quatro Unidades Básicas de Saúde (UBS), com Estratégia Saúde da Família (ESF). Além disso, possui uma Relação Municipal de Medicamentos Essenciais - REMUME, que abrange 125 medicamentos que estão regularmente disponíveis neste estabelecimento e, dos quais, dez se destinam ao tratamento da HAS. A Farmácia Básica é a única que realiza o fornecimento gratuito de medicamentos no município.

Foram incluídos no estudo os usuários, com pelo menos um dos seguintes medicamentos prescritos e dispensados: captopril $25 \mathrm{mg}$, espironolactona $25 \mathrm{mg}$, furosemida $40 \mathrm{mg}$, hidroclorotiazida $25 \mathrm{mg}$, metildopa $250 \mathrm{mg}$, tartarato de metoprolol $100 \mathrm{mg}$, nifedipina 10 e $20 \mathrm{mg}$, propranolol $40 \mathrm{mg}$ e verapamil 80mg. Foram excluídos os indivíduos com déficit cognitivo pela impossibilidade de responder o questionário e gestantes e hemodializados por receberem acompanhamento farmacoterapêutico diferenciado.

O tamanho da amostra foi calculado para contemplar o estudo de prevalência de conhecimento sobre a farmacoterapia da HAS e das associações a serem pesquisadas. Todos os cálculos foram realizados no programa Epi Info $6.04^{33}$. Utilizou-se uma prevalência de conhecimento de $50 \%$, com margem de erro de 4,0 pontos percentuais a um nível de confiança de $95 \%$. Para o estudo das associações, utilizou-se um nível 
de confiança de $95 \%$, poder estatístico de $80 \%$ para preditores com prevalência entre 15 e 85\%, prevalência de conhecimento de $35 \%$ para os não expostos e um risco relativo de 1,5. Os cálculos resultaram em uma amostra necessária de 660 pessoas já acrescidos de 10\% para perdas e 15\% para controle de fatores de confusão.

O trabalho de campo foi desenvolvido no período de quatro de novembro de 2010 a 11 de fevereiro de 2011, tendo a participação de quatro entrevistadoras com ensino médio completo, que receberam um treinamento específico de 20 horas.

Realizou-se um processo de amostragem de forma consecutiva de usuários que acessaram a farmácia no período estudado. Aqueles que preenchiam os critérios de inclusão eram, imediatamente após a dispensação do medicamento, convidados a participar da pesquisa. Após a assinatura do Termo de Consentimento Livre e Esclarecido (TCLE), era realizada a entrevista mediante a aplicação de um questionário padronizado e pré-codificado. O usuário era entrevistado uma única vez ainda que tornasse a utilizar o serviço da farmácia básica no período da investigação. Os sujeitos que referiram não ter tempo para participar da pesquisa eram convidados novamente a cada dispensação realizada no decorrer do estudo. Em caso de recusa, a pesquisadora realizava novamente o convite.

As variáveis sociodemográficas avaliadas foram sexo, idade (coletada em anos completos e posteriormente categorizada), cor da pele (observada e classificada em branca ou não branca pela entrevistadora, uma vez que não era objetivo do estudo detalhar as demais categorias de cor), escolaridade (coletada em anos completos de estudo e analisada em categorias) e situação de moradia (morar só ou não). Como variáveis comportamentais investigou-se o hábito de fumar (categorizado em fumante, ex-fumante e nunca fumou) e a prática de atividade física (categorizada em pratica e não pratica alguma atividade física semanalmente por pelo menos $30 \mathrm{mi}-$ nutos). Como variável de saúde avaliou-se a autopercepção de saúde (excelente, muito boa, boa, regular e ruim, categorizada em boa/muito boa/ excelente e ruim/regular). Foi avaliado, ainda, se o usuário recebeu orientação sobre a farmacoterapia na consulta médica e na dispensação dos medicamentos na farmácia, bem como o número total de medicamentos prescritos (posteriormente categorizado em um, dois, três, quatro e cinco ou mais).

Para definir o desfecho, foi elaborado um questionário que abordava os seguintes aspec- tos sobre o conhecimento dos usuários, para cada um dos dez medicamentos avaliados: nome do medicamento, indicação terapêutica, dose, frequência/horários de administração e duração do tratamento. Antes de dar início à entrevista, as informações da prescrição médica relativas aos medicamentos avaliados eram transcritas para o questionário pela entrevistadora. Durante a entrevista, permitia-se ao usuário consultar a receita, embalagem ou anotações em papel para responder essas questões. O conhecimento foi definido pela concordância entre as respostas dos usuários e as informações contidas na prescrição médica, mesmo quando uma terminologia diferente era utilizada.

A fonte de informação de referência para o julgamento das respostas sobre o nome, dose, frequência/horários de administração foi somente a prescrição médica. Entretanto, para a indicação terapêutica (informação normalmente ausente na prescrição) medida pela pergunta Para que problema de saúde o(a) Sr(a) toma este remédio? foram aceitas além da HAS ou terminologias similares, também outras indicações previstas na bula do medicamento. Nos casos em que a duração do tratamento também não estava presente na prescrição médica e, considerando que os medicamentos avaliados são de uso contínuo, foram aceitas como corretas as respostas "até a próxima consulta" e "por toda a vida". A investigação do conhecimento do usuário sobre o nome do medicamento era realizada de duas formas: primeiro perguntava-se o nome do remédio apontando-o na receita médica e, caso o usuário não soubesse responder, realizava-se a pergunta novamente, apontando para a caixa ou embalagem tipo blister do medicamento respectivo. Este aspecto foi posteriormente categorizado em conhece (na receita ou blister) e não conhece conforme o prescrito. Respostas dadas em nome comercial para medicamentos prescritos pelo nome genérico e vice-versa foram aceitas.

Um escore de conhecimento foi obtido através do número de respostas corretas, com peso igual para cada aspecto avaliado, podendo a pontuação total do escore variar de 0 (zero) a 5 (cinco) para cada um dos dez medicamentos avaliados. Foram atribuídos pesos iguais para cada aspecto da farmacoterapia avaliado, considerando-se que as questões que definiram o escore incluem o conhecimento necessário para o uso seguro e correto de medicamentos utilizados no controle da HAS. Estas perguntas foram testadas e aperfeiçoadas em estudos piloto. Para construir o escore de conhecimento final, a pontua- 
ção total de cada indivíduo foi dividida pelo número de medicamentos prescritos para HAS avaliados no estudo.

Os questionários foram revisados e codificados logo após as entrevistas. A digitação dos dados foi realizada através do programa Epidata 3.0 com checagem automática de consistência e dupla digitação para correção dos possíveis erros de digitação. A análise dos dados foi realizada no programa estatístico Stata 8.0.

A análise descritiva caracterizou os usuários conforme as variáveis sociodemográficas, comportamentais, de saúde, orientação médica e no momento da dispensação e número total de medicamentos prescritos. As perguntas que deram origem ao escore de conhecimento foram também analisadas individualmente e as prevalências de conhecimento descritas. Foram apresentadas as médias, o desvio padrão e a mediana do escore de conhecimento total e para cada medicamento avaliado.

Realizou-se a análise de regressão linear bivariada e análise de variância para testar a associação entre as exposições em estudo e o escore de conhecimento, uma vez que o desfecho apresentou distribuição normal. $\mathrm{Na}$ análise bivariada foi verificada a tendência linear das médias para as variáveis ordinais e realizado o teste de heterogeneidade de médias para as variáveis categóricas. A análise ajustada teve como proposta um modelo hierarquizado que compreende quatro níveis de determinação ${ }^{34}$. No nível mais distal encontram-se as variáveis sociodemográficas: sexo, idade, cor da pele, escolaridade e situação de moradia. No segundo nível foram incluídas as variáveis comportamentais hábito de fumar e prática de atividade física. No terceiro nível encontra-se a variável autopercepção de saúde. As variáveis orientação médica e orientação na dispensação do medicamento além do número total de medicamentos prescritos foram avaliadas como determinantes proximais do conhecimento sobre a farmacoterapia da HAS. Para o controle de fatores de confusão, o efeito de cada preditor foi controlado para as variáveis do mesmo nível ou superior adotando-se um valor $\mathrm{p} \leq 0,2$.

Esta pesquisa foi aprovada pelo Comitê de Ética em Pesquisa da Universidade do Vale dos Sinos. Os participantes assinaram um termo de consentimento para a realização da entrevista. Foi garantido o sigilo quanto à identidade e confidencialidade das informações, bem como o direito de não participar ou suspender a participação a qualquer momento.

\section{Resultados}

De um total de 864 usuários elegíveis, 18 (2,1\%) não aceitaram participar do estudo, 31 (3,6\%) foram excluídos segundo os critérios utilizados e o restante, 137 (15,9\%), foram perdas devido ao tempo de espera na farmácia até a realização da entrevista. Foram entrevistados 678 adultos de 20 anos ou mais de idade, usuários de medicamentos utilizados no tratamento da HAS, dispensados na farmácia básica do município de São Francisco de Paula. A amostra obtida permitiu um poder estatístico de $80 \%$ e um nível de confiança de $95 \%$ para a detecção de diferenças de médias de escore de conhecimento iguais ou maiores que 0,25 ( $\mathrm{dp}=1,00)$.

Como mostra a Tabela 1, os usuários incluídos neste estudo foram predominantemente do sexo feminino (67\%), idade média de 58 anos $(\mathrm{dp}=12,4)$ e metade com primeiro grau incompleto de escolaridade. Do total de pacientes entrevistados, $82 \%$ relataram ter recebido orientação médica sobre como tomar seus medicamentos enquanto $57 \%$ referiu essa orientação na dispensação. Mais da metade da amostra possuía quatro ou mais medicamentos prescritos.

A Tabela 2 apresenta a prevalência de conhecimento da amostra estudada em relação à farmacoterapia para os 10 medicamentos investigados. Entre estes, os mais prescritos foram o captopril $(58,1 \%)$, a hidroclorotiazida $(51,8 \%)$, o propranolol $(17,9 \%)$ a furosemida $(11,7 \%)$ e o metoprolol $(8,0 \%)$. Ao investigar o conhecimento dos usuários sobre o nome do medicamento, observa-se que, entre os medicamentos mais prescritos, os agentes anti-hipertensivos captopril $(88,8 \%)$, propranolol $(74,4 \%)$ e metoprolol $(85,2 \%)$ foram melhor identificados em comparação aos diuréticos hidroclorotiazida $(60,7 \%)$, e Furosemida (62,0\%).

Quando foi pesquisado para qual problema de saúde o medicamento foi receitado, chama atenção que os diuréticos (hidroclorotiazida, furosemida e espironolactona) não foram identificados como prescritos para o tratamento da HAS por mais de $2 / 3$ da amostra. Para este aspecto, a indicação terapêutica correta do captopril e propranolol foi relatada por $95 \%$ e $81 \%$ dos usuários, respectivamente (Tabela 2 ).

O conhecimento sobre a dose correta dos medicamentos avaliados também foi distinto. Para os diuréticos cujo uso recomendado, geralmente, é de uma vez ao dia, mais de $80 \%$ dos usuários conhecem a dose correta; já para medicamentos com posologia mais complexa, como 
Tabela 1. Características dos usuários de medicamentos anti-hipertensivos conforme variáveis sociodemográficas, comportamentais, de saúde, orientação médica e na dispensação dos medicamentos e número de medicamentos. São Francisco de Paula, RS, Brasil, 2010.

\begin{tabular}{|c|c|c|}
\hline Variável & $\mathrm{N}^{\mathrm{a}, \mathrm{b}}$ & $\%$ \\
\hline Total & 678 & 100,0 \\
\hline \multicolumn{3}{|l|}{ Sexo } \\
\hline Masculino & 224 & 33,0 \\
\hline Feminino & 454 & 67,0 \\
\hline \multicolumn{3}{|l|}{ Idade (anos) } \\
\hline$\leq 44$ & 97 & 14,3 \\
\hline $45-54$ & 169 & 24,9 \\
\hline $55-64$ & 177 & 26,1 \\
\hline$\geq 65$ & 235 & 34,7 \\
\hline \multicolumn{3}{|l|}{ Cor da pele } \\
\hline Branca & 493 & 72,7 \\
\hline Não branca & 185 & 27,3 \\
\hline \multicolumn{3}{|l|}{ Escolaridade (anos completos) } \\
\hline 0 & 100 & 14,7 \\
\hline $1-4$ & 237 & 35,0 \\
\hline $5-8$ & 246 & 36,3 \\
\hline 9 ou mais & 95 & 14,0 \\
\hline \multicolumn{3}{|l|}{ Situação de moradia } \\
\hline Mora só & 102 & 15,1 \\
\hline Não mora só & 573 & 84,9 \\
\hline \multicolumn{3}{|l|}{ Hábito de fumar } \\
\hline Fumante & 141 & 20,8 \\
\hline Ex-fumante & 219 & 32,3 \\
\hline Nunca fumou & 318 & 46,9 \\
\hline \multicolumn{3}{|l|}{ Atividade física } \\
\hline Pratica & 193 & 28,6 \\
\hline Não pratica & 483 & 71,4 \\
\hline \multicolumn{3}{|l|}{ Autopercepção de saúde } \\
\hline Boa/ Muito boa/ Excelente & 241 & 35,6 \\
\hline Regular/Ruim & 435 & 64,4 \\
\hline \multicolumn{3}{|l|}{ Orientação médica } \\
\hline Sim & 559 & 82,5 \\
\hline Não & 119 & 17,5 \\
\hline \multicolumn{3}{|l|}{ Orientação na dispensação } \\
\hline Sim & 389 & 57,4 \\
\hline Não & 289 & 42,6 \\
\hline \multicolumn{3}{|l|}{$\begin{array}{l}\text { Numero total de medicamentos } \\
\text { prescritos }\end{array}$} \\
\hline 1 & 54 & 8,0 \\
\hline 2 & 146 & 21,5 \\
\hline 3 & 116 & 17,1 \\
\hline 4 & 126 & 18,6 \\
\hline 5 ou mais & 236 & 34,8 \\
\hline
\end{tabular}

${ }^{\mathrm{a}}$ Número de pessoas; ${ }^{\mathrm{b}} \mathrm{O}$ número máximo de valores ignorados foi de 3 para situação de moradia.

captopril e propranolol, cerca de 70\% dos usuários conhecem. O mesmo ocorreu em relação à frequência e horários de administração, onde $40 \%$ dos usuários de captopril e propranolol não souberam informar corretamente, enquanto a falta desse conhecimento foi identificada em $20 \%$ dos usuários de diuréticos (Tabela 2).

O conhecimento sobre a duração do tratamento se mostrou deficiente para todos os medicamentos avaliados, uma vez que cerca de 50\% dos usuários não sabiam por quanto tempo deviam tomar os seus medicamentos (Tabela2).

A Tabela 3 apresenta a média do escore de conhecimento total e conforme os medicamentos avaliados. A média de escore de conhecimento sobre a farmacoterapia da HAS na amostra foi de 3,27 ( $\mathrm{dp}=1,16)$. Maiores médias foram observadas em relação aos agentes anti-hipertensivos captopril $(3,75 ; \mathrm{dp}=1,8)$, nifedipina $20 \mathrm{mg}(3,56 ; \mathrm{dp}=1,5)$ e metroprolol $(3,50 ; \mathrm{dp}=$ $1,2)$, enquanto os diuréticos hidroclorotiazida $(3,09 ; \mathrm{dp}=1,26)$ espironolactona $(3,05 ; \mathrm{dp}=1,2)$ e furosemida $(2,92 ; \mathrm{dp}=1,4)$ apresentaram menores médias de escore de conhecimento sobre aspectos da farmacoterapia.

A Tabela 4 apresenta a análise bruta e ajustada entre os fatores investigados e o escore de conhecimento sobre a farmacoterapia da HAS. Após ajuste para fatores de confusão, a idade esteve inversamente associada ao escore de conhecimento ( $p=0,004)$. O oposto ocorreu com a escolaridade que apresentou uma associação linear direta, sendo que usuários com nove anos ou mais de estudo têm um acréscimo de 0,8 na média de escore quando comparados àqueles sem escolaridade ( $\mathrm{p}<0,001)$. Os usuários de cor da pele branca e os que avaliaram a sua saúde como boa/muito boa/excelente apresentaram um escore de conhecimento maior ( $\beta=0,28$; IC95\%: 0,09-0,47, $p=0,005 ; \beta=0,20$; IC95\%: 0,02-0,38, $\mathrm{p}=0,030)$. Observou-se uma associação linear inversa entre número total de medicamentos prescritos e escore de conhecimento $(p=0,014)$.

O diagnóstico do modelo de regressão linear foi realizado por meio do gráfico normal de resíduos e de resíduos padronizados, não apresentando desvio da linearidade.

\section{Discussão}

O controle dos níveis pressóricos é, em sua maioria, realizado com medicamentos. Apesar disso, o presente estudo observou um conhecimento limitado dos portadores de HAS sobre a sua farmacoterapia, para todos os aspectos avaliados. A média do escore de conhecimento observada foi de 3,27 $(\mathrm{dp}=1,16)$. Menor idade, maior 
Tabela 2. Prevalência de conhecimento sobre a farmacoterapia em usuários de medicamentos anti-hipertensivos $(\mathrm{n}=678)$. São Francisco de Paula, RS, Brasil, 2010.

\begin{tabular}{|c|c|c|c|c|c|c|c|c|c|c|}
\hline \multirow[b]{2}{*}{ Variável } & \multicolumn{10}{|c|}{ Medicamentos $^{a}$} \\
\hline & $\begin{array}{c}1 \\
\mathbf{N}=394 \\
\%\end{array}$ & $\begin{array}{c}2 \\
\mathbf{N}=351 \\
\%\end{array}$ & $\begin{array}{c}3 \\
\mathbf{N}=121 \\
\%\end{array}$ & $\begin{array}{c}\mathbf{4} \\
\mathbf{N}=79 \\
\%\end{array}$ & $\begin{array}{c}5 \\
\mathbf{N}=54 \\
\%\end{array}$ & $\begin{array}{c}\mathbf{6} \\
\mathbf{N}=21 \\
\%\end{array}$ & $\begin{array}{c}7 \\
\mathbf{N}=19 \\
\%\end{array}$ & $\begin{array}{c}\mathbf{8} \\
\mathbf{N}=16 \\
\%\end{array}$ & $\begin{array}{c}9 \\
\mathbf{N}=13 \\
\%\end{array}$ & $\begin{array}{c}10 \\
\mathrm{~N}=13 \\
\%\end{array}$ \\
\hline Total & 58,1 & 51,8 & 17,9 & 11,7 & 8,0 & 3,1 & 2,8 & 2,4 & 1,9 & 1,9 \\
\hline Nome do medicamento & 88,8 & 60,7 & 74,4 & 62,0 & 85,2 & 57,2 & 52,6 & 56,3 & 68,8 & 84,6 \\
\hline Receita & 69,8 & 47,3 & 60,3 & 55,7 & 75,9 & 52,4 & 36,8 & 43,8 & 53,4 & 69,2 \\
\hline Blister & 19,0 & 13,4 & 14,1 & 6,3 & 9,3 & 4,8 & 15,8 & 12,5 & 15,4 & 15,4 \\
\hline Indicação & 94,9 & 32,2 & 81,0 & 20,2 & 59,3 & 57,1 & 26,3 & 87,5 & 69,2 & 46,2 \\
\hline Dose & 74,9 & 87,8 & 67,8 & 83,8 & 77,8 & 80,9 & 84,2 & 81,2 & 76,9 & 61,5 \\
\hline Frequência/horário & 60,4 & 82,6 & 60,3 & 78,8 & 79,6 & 71,4 & 79,0 & 75,0 & 69,2 & 61,5 \\
\hline Duração do tratamento & 56,4 & 46,2 & 43,0 & 48,8 & 48,2 & 47,6 & 63,2 & 56,2 & 30,8 & 46,2 \\
\hline
\end{tabular}

${ }^{a}$ Medicamentos ordenados segundo a frequência: 1 = Captopril 25mg; 2 = Hidroclorotiazida 25mg; 3 = Propranolol 40mg. 4 = Furosemida 40mg; 5 = Metoprolol 100mg; 6 = Verapamil 80mg; 7 = Espironolactona 25mg; 8 = Nifedipina 20mg; 9 = Metildopa 250mg; 10 = Nifedipina 10mg.

Tabela 3. Média e mediana do escore de conhecimento total e conforme os medicamentos avaliados em usuários de medicamentos anti-hipertensivos. São Francisco de Paula, RS, Brasil, 2010.

\begin{tabular}{|c|c|c|c|}
\hline Medicamentos $^{\mathrm{a}}$ & Média (desvio padrão) & Mediana & (intervalo interquartil) \\
\hline Captopril 25mg & $3,75(1,19)$ & & $4,0(3,0-5,0)$ \\
\hline Nifedipina 20mg & $3,56(1,46)$ & & $4,0(2,5-5,0)$ \\
\hline Metoprolol 100mg & $3,50(1,22)$ & & $4,0(3,0-4,0)$ \\
\hline Propranolol 40mg & $3,26(1,40)$ & & $4,0(2,0-4,0)$ \\
\hline Metildopa 250mg & $3,15(1,68)$ & & $3,0(3,0-4,0)$ \\
\hline Verapamil $80 \mathrm{mg}$ & $3,14(1,52)$ & & $3,0(2,0-5,0)$ \\
\hline Hidroclorotiazida $25 \mathrm{mg}$ & $3,09(1,26)$ & & $3,0(2,0-4,0)$ \\
\hline Espironolactona $25 \mathrm{mg}$ & $3,05(1,22)$ & & $3,0(2,0-4,0)$ \\
\hline Nifedipina $10 \mathrm{mg}$ & $3,00(1,73)$ & & $3,0(1,0-4,0)$ \\
\hline Furosemida 40mg & $2,92(1,39)$ & & $3,0(2,0-4,0)$ \\
\hline Total & $3,27(1,16)$ & & $3,0(2,0-4,0)$ \\
\hline
\end{tabular}

${ }^{a}$ Medicamentos ordenados segundo a média do escore de conhecimento.

escolaridade, cor da pele branca, melhor autopercepção de saúde e menor número de medicamentos prescritos mostraram-se associados a um maior escore de conhecimento. Quanto aos aspectos que constituíram o escore, percebe-se que o conhecimento sobre o nome do medicamento e a indicação terapêutica foi superior quando avaliados os anti-hipertensivos em comparação aos diuréticos. Contudo, quando foi investigada a dose e a frequência/horários de administração, essa relação se mostrou inversa, uma vez que, as maiores prevalências de conhecimento foram observadas para os diuréticos em relação aos antihipertensivos. Para a duração do tratamento o conhecimento foi similar para todos os medica- mentos avaliados, sendo que somente metade dos usuários referiu corretamente.

Analisando cada aspecto do escore percebese que, em relação ao nome do medicamento, a literatura revela dados semelhantes ${ }^{21,25,30}$. Sabese para condições crônicas como a HAS, frequentemente, são prescritos vários medicamentos e a incapacidade de nomeá-los pode aumentar o risco de erros na administração ou comprometer a adesão ao tratamento ${ }^{20}$. Além disso, no Sistema Único de Saúde (SUS), os medicamentos são, normalmente, dispensados na forma de blister, cuja embalagem e cor do medicamento podem variar conforme o laboratório fornecedor. Assim, é de extrema importância que o usuário sai- 
Tabela 4. Fatores associados ao escore de conhecimento sobre a farmacoterapia em usuários de medicamentos anti-hipertensivos. São Francisco de Paula, RS, Brasil, 2010.

\begin{tabular}{|c|c|c|c|c|c|c|}
\hline & \multirow[b]{2}{*}{ Variável } & \multirow[b]{2}{*}{$\begin{array}{c}\text { Média } \\
\text { (Desvio } \\
\text { Padrão) }\end{array}$} & \multicolumn{2}{|l|}{ Análise bruta } & \multicolumn{2}{|c|}{ Análise Ajustada ${ }^{e}$} \\
\hline & & & $\begin{array}{c}\beta^{\mathrm{c}} \\
\left(\mathrm{I} \mathrm{C}^{\mathrm{d}} \mathbf{9 5 \%}\right)\end{array}$ & Valor $\mathrm{p}$ & $\begin{array}{c}\beta^{\mathrm{c}} \\
\left(\mathrm{IC}^{\mathrm{d}} \mathbf{9 5 \%}\right)\end{array}$ & Valor $\mathbf{p}$ \\
\hline \multirow{19}{*}{ 总 } & Sexo ${ }^{f}$ & & & $0,670^{\mathrm{b}}$ & & $0,302^{\mathrm{b}}$ \\
\hline & Masculino & $3,16(1,27)$ & & & & \\
\hline & Feminino & $3,33(1,11)$ & $0,17(-0,01 ; 0,36)$ & & $0,10(-0,09 ; 0,29)$ & \\
\hline & Idade & & & $<0,001^{\mathrm{a}}$ & & $0,004^{\mathrm{a}}$ \\
\hline & $\leq 44$ anos & $3,48(1,16)$ & $0,40(0,13 ; 0,68)$ & & $0,30(0,02 ; 0,57)$ & \\
\hline & $45-54$ anos & $3,52(1,04)$ & $0,44(0,21 ; 0,67)$ & & $0,32(0,09 ; 0,55)$ & \\
\hline & $55-64$ anos & $3,18(1,18)$ & $0,09(-0,13 ; 0,32)$ & & $0,05(-0,18 ; 0,27)$ & \\
\hline & $\geq 65$ anos & $3,08(1,20)$ & & & & \\
\hline & Cor da pele & & & $0,002^{\mathrm{b}}$ & & $0,005^{\mathrm{b}}$ \\
\hline & Branca & $3,36(1,10)$ & $0,32(0,12 ; 0,51)$ & & $0,28(0,09 ; 0,47)$ & \\
\hline & Não branca & $3,04(1,29)$ & & & & \\
\hline & Escolaridade (anos de estudo) & & & $<0,001^{\mathrm{a}}$ & & $<0,001^{\mathrm{a}}$ \\
\hline & 0 & $2,65(1,21)$ & & & & \\
\hline & $1-4$ & $3,28(1,15)$ & $0,63(0,36 ; 0,89)$ & & $0,55(0,29 ; 0,82)$ & \\
\hline & $5-8$ & $3,41(1,09)$ & $0,76(0,50 ; 1,03)$ & & $0,64(0,37 ; 0,91)$ & \\
\hline & $9 \mathrm{ou}+$ & $3,55(1,13)$ & $0,90(0,58 ; 1,22)$ & & $0,76(0,44 ; 1,08)$ & \\
\hline & Situação de moradia ${ }^{\mathrm{f}}$ & & & $0,352^{\mathrm{b}}$ & & $0,933^{\mathrm{b}}$ \\
\hline & Mora só & $3,18(1,25)$ & $0,00(-0,01 ; 0,00)$ & & $0,00(-0,01 ; 0,01)$ & \\
\hline & Não mora só & $3,29(1,15)$ & & & & \\
\hline \multirow{7}{*}{$\frac{N}{2}$} & Hábito de fumar ${ }^{\mathrm{f}}$ & & & $0,298^{\mathrm{b}}$ & & $0,295^{\mathrm{b}}$ \\
\hline & Fumante & $3,36(1,24)$ & & & & \\
\hline & Ex-fumante & $3,18(1,15)$ & $-0,18(-0,43 ; 0,06)$ & & $-0,18(-0,42 ; 0,06)$ & \\
\hline & Não fumante & $3,30(1,14)$ & $-0,06(-0,29 ; 0,17)$ & & $-0,06(-0,29 ; 0,16)$ & \\
\hline & Atividade física ${ }^{\mathrm{f}}$ & & & $0,166^{\mathrm{b}}$ & & $0,211^{\mathrm{b}}$ \\
\hline & Pratica & $3,17(1,09)$ & $-0,14(-0,33 ; 0,06)$ & & $-0,12(-0,31 ; 0,07)$ & \\
\hline & Não pratica & $3,31(1,19)$ & & & & \\
\hline \multirow{3}{*}{$\frac{n}{\tilde{z}}$} & Autopercepção de Saúde & & & $0,003^{\mathrm{b}}$ & & $0,030^{\mathrm{b}}$ \\
\hline & Boa/ Muito boa/ Excelente & $3,45(1,04)$ & $0,27(0,09 ; 0,46)$ & & $0,20(0,02 ; 0,38)$ & \\
\hline & Regular/Ruim & $3,17(1,22)$ & & & & \\
\hline \multirow{12}{*}{$\frac{+}{\square}$} & Orientação médica & & & $0,013^{\mathrm{b}}$ & & $0,113^{\mathrm{b}}$ \\
\hline & Sim & $3,32(1,11)$ & $0,29(0,06 ; 0,52)$ & & $0,18(-0,04 ; 0,41)$ & \\
\hline & Não & $3,03(1,36)$ & & & & \\
\hline & Orientação na dispensação ${ }^{f}$ & & & $0,458^{\mathrm{b}}$ & & $0,870^{\mathrm{b}}$ \\
\hline & $\operatorname{Sim}$ & $3,24(1,20)$ & $-0,07(-0,24 ; 0,11)$ & & $-0,01(-0,19 ; 0,16)$ & \\
\hline & Não & $3,31(1,11)$ & & & & \\
\hline & Número total de medicamentos prescritos & & & $<0,001^{\mathrm{a}}$ & & $0,014^{\mathrm{a}}$ \\
\hline & 1 & $3,68(1,23)$ & $0,63(0,29 ; 0,98)$ & & $0,41(0,07 ; 0,76)$ & \\
\hline & 2 & $3,40(1,08)$ & $0,35(0,11 ; 0,58)$ & & $0,19(-0,05 ; 0,43)$ & \\
\hline & 3 & $3,40(1,11)$ & $0,35(0,09 ; 0,60)$ & & $0,22(-0,03 ; 0,48)$ & \\
\hline & 4 & $3,25(1,12)$ & $0,20(-0,05 ; 0,45)$ & & $0,12(-0,13 ; 0,36)$ & \\
\hline & 5 ou mais & $3,05(1,21)$ & & & & \\
\hline
\end{tabular}

${ }^{\mathrm{a}}$ Teste de Wald para tendência linear; ${ }^{\mathrm{b}}$ Teste de Wald para heterogeneidade de médias; ${ }^{\mathrm{c}}$ Coeficiente $\beta ;{ }^{\mathrm{d}}$ Intervalo de Confiança de $95 \% ;{ }^{\mathrm{e}}$ Cada variável está ajustada para as do mesmo nível e as dos níveis acima (foram mantidas no modelo as variáveis com valor $\mathrm{p} \leq 0,2$ ); ‘ $\mathrm{f}$ exo, situação de moradia, hábito de fumar, atividade física e orientação na dispensação não se mantiveram no modelo final $(p>0,2)$. 
ba nomear o medicamento para identificá-lo também nessas situações.

Quanto à indicação terapêutica a literatura revela um melhor nível de conhecimento sobre esse aspecto com relação aos diuréticos. Estudos realizados na Escócia e nos Estados Unidos verificaram que mais de 70\% dos usuários de diuréticos conheciam a indicação correta destes medicamentos $^{28,30}$. No presente estudo, somente $1 / 3$ dos usuários souberam informar corretamente. Quanto à indicação terapêutica dos anti-hipertensivos os dados concordam com a literatura, revelando prevalências maiores do que as encontradas para os diuréticos $22,25,29,31,32$. Estes resultados são preocupantes, uma vez que os usuários portadores de HAS podem interromper o uso de diuréticos pelos efeitos que eles causam se não relacioná-los a manutenção dos níveis pressóricos. O escasso conhecimento sobre a indicação dos diuréticos no controle da HAS pode indicar também que a informação fornecida na consulta médica não tem contemplado esse aspecto.

Apesar da elevada prevalência de respostas corretas sobre a dose e a frequência/horários de administração, os resultados apontam falhas no conhecimento para medicamentos com posologia mais complexa em comparação aqueles, normalmente prescritos para o uso uma vez ao dia. Poucos estudos avaliaram estes aspectos para os anti-hipertensivos e encontraram prevalências superiores em relação aos betabloqueadores ${ }^{22,29}$ enquanto para os diuréticos, Bevan et al. ${ }^{28}$ e Gibbs et al. ${ }^{30}$ encontraram prevalências semelhantes'. Estudo nacional realizado por Silva et al. ${ }^{35}$ também verificou prevalências semelhantes de conhecimento sobre a dose e frequência/horários de administração quando avaliou medicamentos de uso contínuo e eventual. O conhecimento sobre estes aspectos é importante, pois, mesmo com um diagnóstico e uma escolha terapêutica adequada a efetividade do tratamento pode estar comprometida se o usuário não souber como tomar seus medicamentos. Como consequência, uma dose menor que o recomendado pode prejudicar a efetividade do medicamento e, por outro lado, ao tomar uma dose maior que a necessária, o usuário poderá experimentar efeitos adversos ao medicamento.

A duração do tratamento foi o aspecto da farmacoterapia que apresentou menor conhecimento. Estudos revelam resultados distintos mostrando que o conhecimento sobre a duração do tratamento depende mais da forma como foi avaliado do que das características sociodemográficas das populações estudadas. Mesmo es- tudos nacionais com populações semelhantes mostraram resultados superiores aos encontrados neste estudo, onde mais de 70\% dos usuários sabiam que o tratamento da HAS é para toda vida ou contínuo ${ }^{16,18,23,24,27}$. Entretanto, o conhecimento foi medido pela afirmativa: "O tratamento da Hipertensão é para toda vida”, o que pode ter induzido o entrevistado a responder afirmativamente. Vale salientar que o conhecimento do usuário sobre o tempo de uso do medicamento está diretamente associado à adesão ao tratamento ${ }^{25}$, pois o torna consciente de que seus níveis pressóricos estão normalizados pelo uso do medicamento e não pela cura da HAS, sendo assim possui um papel fundamental no controle desta morbidade.

A média do escore observada $(3,27)$ correspondeu a $65,4 \%$ do total de acertos. Na literatura, localizou-se um único estudo com metodologia semelhante, o qual encontrou um escore de conhecimento inferior $(62,0 \%)$ para uma pontuação menor (0-4), avaliando medicamentos dispensados para uso contínuo e eventual ${ }^{36}$. Os resultados do presente estudo revelam um conhecimento limitado, considerando que foram investigados aspectos necessários para o uso correto e seguro dos medicamentos avaliados.

Conforme a literatura revisada, não foram identificados estudos com metodologia semelhante à proposta nesta pesquisa quanto aos medicamentos investigados, população avaliada e fatores associados ao conhecimento, analisado como um escore. Assim, as comparações a seguir serão feitas a luz dessas limitações.

Analisando os fatores associados ao escore de conhecimento, observou-se que o aumento da idade esteve associado ao menor escore de conhecimento sobre a farmacoterapia. Esta associação é consistente com outros estudos mesmo com metodologias distintas e diferentes grupos farmacológicos investigados ${ }^{28,31,32,36-39}$. Sabese que usuários com maior idade podem apresentar dificuldade de memorização. Além disso, com o aumento da idade também ocorre um aumento do número de medicamentos prescritos, dificultando ainda mais a capacidade de armazenar corretamente as informações.

Neste estudo, a escolaridade esteve diretamente associada ao escore de conhecimento. Estes dados são consistentes com outras pesquisas realizadas no Brasil $^{40}$ e na população mundi$\mathrm{al}^{24,31,37,38}$. Está bem estabelecido que a maior escolaridade é preditora de um maior conhecimento, independente do aspecto sendo avaliado. Além disso, ela pode contribuir para a interação 
do usuário com o profissional de saúde durante a consulta médica ou dispensação do medicamento, no que diz respeito as suas necessidades em saúde.

Quanto à cor da pele, o estudo mostrou que usuários brancos apresentam maior escore de conhecimento quando comparados a usuários não brancos. Essa relação também foi observada por Persell et al..$^{31}$, que verificaram que usuários de cor da pele não branca possuem duas vezes mais chance de não conhecerem a indicação terapêutica de medicamentos de uso contínuo quando comparados àqueles de cor da pele branca. Este resultado pode estar relacionado à iniquidade dos cuidados em saúde com relação a indivíduos brancos e não brancos, uma vez que brancos recebem uma melhor atenção médica, conforme apontam estudos que avaliaram a realização de exames clínicos de mama e citopatológico ${ }^{41,42}$.

Este estudo também mostrou que usuários que percebem melhor a sua saúde apresentaram um maior escore de conhecimento. Essa associação não foi observada no estudo americano realizado por Viera et al. ${ }^{24}$ quanto à duração do tratamento. No entanto, indivíduos que percebem melhor sua saúde podem apresentar atitudes mais positivas e, por isso, buscar mais informações sobre os cuidados em saúde.

Outro preditor de um maior escore de conhecimento foi o número total de medicamentos prescritos, apresentando uma associação inversa. Este achado é evidenciado na literatura, tanto em estudos que incluem medicamentos de uso contínuo em sua análise $e^{28,32,38,43}$ como aqueles que avaliam medicamentos de uso eventual ${ }^{31,36}$. É plausível pensar que indivíduos com menor conhecimento sobre a sua farmacoterapia cuidem menos adequadamente de sua saude e, consequentemente, necessitem utilizar maior número de medicamentos. Entretanto, deve-se considerar que este estudo utilizou um delineamento transversal que não permite verificar relações de causa e efeito, uma vez que as informações sobre exposição e desfecho são coletadas no mesmo momento. Este aspecto pode ter afetado algumas exposições como o número de medicamentos e o escore de conhecimento.

Entre os fatores investigados, a orientação médica e a orientação realizada durante a dispensação dos medicamentos não estiveram associados ao escore de conhecimento. Para o primeiro, pode-se atribuir a redução do poder estatístico após ajuste do modelo. Entretanto, ambos os resultados podem estar refletindo a qua- lidade das informações fornecidas aos usuários durante a consulta médica e a dispensação dos medicamentos. Estudos mostram que o tempo médio destinado à consulta médica e à dispensação de medicamentos no setor público impossibilita uma orientação adequada aos usuários ${ }^{44,45}$. Em contrapartida, estudos de intervenção relatam uma melhora significativa no conhecimento dos usuários que receberam orientação verbal ou escrita em comparação aos que não receberam esta intervenção ${ }^{11,46}$. Estas evidências apontam para a necessidade de estratégias educacionais a serem desenvolvidas pelos profissionais da área da saúde, visando apoiar o usuário no processo de uso de seus medicamentos. Entre estes, está o farmacêutico que constitui o último contato do usuário com o sistema de saúde antes do início do processo de uso do medicamento. $\mathrm{O}$ farmacêutico deve assumir sua responsabilidade na conclusão do ciclo da assistência farmacêutica, o qual termina com a dispensação ativa. Esta deve considerar as necessidades dos usuários no processo de uso do medicamento, promovendo ainda o acompanhamento farmacoterapêutico além de todas as informações necessárias para que o usuário realize corretamente o seu tratamento. Portanto, ações simples, como a realização de prescrições legíveis que contenham todas as informações necessárias para o uso correto da farmacoterapia, o reforço verbal das orientações realizadas durante a consulta médica e a dispensação na farmácia, incluindo a informação de como os medicamentos prescritos atuam no controle da HAS, podem contribuir para a utilização dos medicamentos conforme a prescrição.

Em conclusão, o estudo revela que o conhecimento que o usuário da atenção básica possui sobre o tratamento farmacológico da HAS ainda é insuficiente, podendo comprometer o uso correto e seguro dos medicamentos. $\mathrm{O}$ estudo aponta também a necessidade de melhor orientação e acompanhamento dos usuários idosos, com menor escolaridade, não brancos e com maior número de medicamentos prescritos para o controle dessa morbidade. Por fim, deve-se considerar que o acesso gratuito a medicamentos tem sido garantido por vários programas, principalmente para usuários de medicamentos destinados ao tratamento da HAS, que dependem do setor público para obtê-los. Apesar disso, grande parte deles não sabe como utilizar corretamente seus medicamentos, o que coloca em risco a efetividade do tratamento prescrito contrariando as políticas de uso racional de medicamentos. 


\section{Colaboradores}

FR Motter revisou a literatura, elaborou o projeto e o instrumento de coleta de dados, realizou o treinamento das entrevistadoras, supervisionou o trabalho de campo, trabalhou na análise dos dados e na redação do artigo. MTA Olinto participou da elaboração do projeto e do instrumento de coleta de dados, análise dos dados e redação do artigo final. VMV Paniz coordenou todas as etapas do projeto, da concepção, delineamento, análise dos dados até a redação final do artigo. Todos os autores trabalharam na redação do artigo, revisaram criticamente e aprovaram a versão final.

\section{Agradecimentos}

Ao Conselho Nacional de Desenvolvimento Científico e Tecnológico pelo apoio financeiro.

\section{Referências}

1. Chobanian AV, Bakris GL, Black HR, Cushman WC, Green LA, Izzo Júnior JL, Jones DW, Materson BJ, Oparil S, Wright Júnior JT, Roccella EJ. The Seventh Report of the Joint National Committee on Prevention, Detection, Evaluation, and Treatment of High Blood Pressure: the JNC 7 report. JAMA 2003; 289(19):2560-2572.

2. Lewington S, Clarke R, Qizilbash N, Peto R, Collins R. Age-specific relevance of usual blood pressure to vascular mortality: a meta-analysis of individual data for one million adults in 61 prospective studies. Lancet 2002; 360(9349):1903-1913.

3. Cipullo JP, Martin JF, Ciorlia LA, Godoy MR, Cacao JC, Loureiro AA, Cesarino CB, Carvalho AC, Cordeiro JA, Burdmann Ede A. Prevalência e Fatores de Risco para Hipertensão em uma População Urbana Brasileira. Arq Bras Cardiol 2010; 94(4):519-526.

4. Longo GZ, Neves J, Luciano VM, Peres MA. Prevalência de níveis Pressóricos Elevados e Fatores Associados em Adultos de Lages/SC. Arq Bras Cardiol 2009; 93(4):387-394.

5. Chrestani MA, Santos Ida S, Matijasevich AM. Hipertensão arterial sistêmica auto-referida: validação diagnóstica em estudo de base populacional. Cad Saude Publica 2009; 25(11):2395-2406.

6. Rosario TM, Scala LC, Franca GV, Pereira MR, Jardim PC. Prevalência, controle e tratamento da hipertensão arterial sistêmica em Nobres - MT. Arq Bras Cardiol 2009; 93(6):622-528.

7. Sociedade Brasileira de Cardiologia (SBC), Sociedade Brasileira de Hipertensão (SBH), Sociedade Brasileira de Nefrologia (SBN). VI Diretrizes Brasileiras de Hipertensão. Arq Bras Cardiol 2010. 95(Supl. 1):1-51.

8. Dias-da-Costa JS, Barcellos FC, Sclowitz ML, Sclowitz IK, Castanheira M, Olinto MT, Menezes AM, Gigante DP, Macedo S, Fuchs SC. Prevalência de hipertensão arterial em adultos e fatores associados: um estudo de base populacional urbana em Pelotas, Rio Grande do Sul, Brasil. Arq Bras Cardiol 2007; 88(1):59-65.

9. Nogueira D, Faerstein E, Coeli CM, Chor D, Lopes C de S, Werneck GL. Reconhecimento, tratamento e controle da hipertensão arterial: estudo Pró-Saúde, Brasil. Rev Panam Salud Publica 2010; 27(2):103-109.

10. Brasil. Ministério da Saúde (MS). Secretaria de Políticas de Saúde. Departamento de Ações Programáticas Estratégicas. Plano de reorganização da atenção à hipertensão arterial e ao diabetes mellitus: hipertensão arterial e diabetes mellitus. Brasília: MS; 2001. [Série C. Projetos, Programas e Relatórios, No 59]

11. Ponnusankar S, Surulivelrajan M, Anandamoorthy N, Suresh B. Assessment of impact of medication counseling on patients' medication knowledge and compliance in an outpatient clinic in South India. Patient Educ Couns 2004; 54(1):55-60.

12. Okuno J, Yanagi H, Tomura S, Oka M, Hara S, Hirano C, Tsuchiya S. Compliance and medication knowledge among elderly Japanese home-care recipients. Eur J Clin Pharmacol 1999; 55(2):145-149. 
13. Persell SD, Bailey SC, Tang J, Davis TC, Wolf MS. Medication reconciliation and hypertension control. Am J Med 2010; 123(2):182 e9-e15.

14. Morgado M, Rolo S, Macedo AF, Pereira L, Castelo-Branco M. Predictors of uncontrolled hypertension and antihypertensive medication nonadherence. J Cardiovasc Dis Res 2010; 1(4):196-202.

15. Mion Júnior D, Pierin AM, Bensenor IM, Marin JC, Costa KR, Henrique LF, Alencar NP, Couto RC, Laurenti TE, Machado TA. Hipertensão arterial na cidade de São Paulo: prevalência referida por contato telefônico. Arq Bras Cardiol 2010; 95(1):99-106.

16. Cuspidi C, Lonati L, Sampieri L, Michev I, Macca G, Fusi V, Salerno M, Zanchetti A. "To better know hypertension": educational meetings for hypertensive patients. Blood Press 2000; 9(5):255-259.

17. Cuspidi C, Sampieri L, Macca G, Michev I, Fusi V, Salerno M, Severgnini B, Rocanova JI, Leonetti G, Zanchetti A. Improvement of patients' knowledge by a single educational meeting on hypertension. $J$ Hum Hypertens 2001; 15(1):57-61.

18. Strelec MA, Pierin AM, Mion Júnior D. The influence of patient's consciousness regarding high blood pressure and patient's attitude in face of disease controlling medicine intake. Arq Bras Cardiol 2003; 81(4):349-354.

19. Familoni BO, Ogun SA, Aina AO. Knowledge and awareness of hypertension among patients with systemic hypertension. J Natl Med Assoc 2004; 96(5):620624.

20. Persell SD, Osborn CY, Richard R, Skripkauskas S, Wolf MS. Limited health literacy is a barrier to medication reconciliation in ambulatory care. $J$ Gen Intern Med 2007; 22(11):1523-1526.

21. Ragot S, Sosner P, Bouche G, Guillemain J, Herpin D. Appraisal of the knowledge of hypertensive patients and assessment of the role of the pharmacists in the management of hypertension: results of a regional survey. J Hum Hypertens 2005; 19(7):577-584.

22. Gibbs S, Waters WE, George CF. The benefits of prescription information leaflets (1). Br J Clin Pharmacol 1989; 27(6):723-739.

23. Sanchez CG, Pierin AM, Mion Junior D. Comparison of the profiles of hypertensive patients seen at the emergency department and in ambulatory care. Rev Esc Enferm USP 2004; 38(1):90-98.

24. Viera AJ, Cohen LW, Mitchell CM, Sloane PD. High blood pressure knowledge among primary care patients with known hypertension: a North Carolina Family Medicine Research Network (NC-FM-RN) study. J Am Board Fam Med 2008; 21(4):300-308.

25. Karaeren H, Yokusoglu M, Uzun S, Baysan O, Koz C, Kara B, Kirilmaz A, Naharci I, Pinar M, Yilmaz MB, Uzun M. The effect of the content of the knowledge on adherence to medication in hypertensive patients. Anadolu Kardiyol Derg 2009; 9(3):183-188.

26. Fagerlin A, Sepucha KR, Couper MP, Levin CA, Singer E, Zikmund-Fisher BJ. Patients' knowledge about 9 common health conditions: the DECISIONS survey. Med Decis Making 2010; 30(Supl. 5):35S-52S
27. Chai Y, Xu H, Wang W, Liu B, Yang D, Fan H, Song F, Lu Z. A survey of factors associated with the utilization of community health centers for managing hypertensive patients in Chengdu, China. PLoS One 2011; 6(7):e217-e218.

28. Bevan EG, Currie EM, McGhee SM, McInnes GT. Patient knowledge about diuretic prescription. $\mathrm{Br} J$ Clin Pharmacol 1993; 35(2):152-155.

29. Nurit P, Bella BC, Gila E, Revital Z. Evaluation of a nursing intervention project to promote patient medication education. J Clin Nurs 2009; 18(17):25302536.

30. Gibbs S, Waters WE, George CF. The benefits of prescription information leaflets (2). Br J Clin Pharmacol 1989; 28(3):345-351.

31. Persell SD, Heiman HL, Weingart SN, Burdick E, Borus JS, Murff HJ, Bates DW, Gandhi TK. Understanding of drug indications by ambulatory care patients. Am J Health Syst Pharm 2004; 61(23):25232527.

32. Micheli P, Kossovsky MP, Gerstel E, Louis-Simonet M, Sigaud P, Perneger TV, Gaspoz, JM. Patients' knowledge of drug treatments after hospitalisation: the key role of information. Swiss Med Wkly 2007; 137(43-44):614-620.

33. Dean AG DJ, Colombier D, Brendel KA, Smith DC, Burton AH, Dicker RC, Sullivan K, Fagan RF, Arner TG. EpiInfo Version 6.04: a word processing database and statistics program for epidemiology. Atlanta: Center Disease Control and Prevention; 2001.

34. Victora CG, Huttly SR, Fuchs SC, Olinto MT. The role of conceptual frameworks in epidemiological analysis: a hierarchical approach. Int J Epidemiol 1997; 26(1):224-227.

35. Silva T, Schenkel EP, Mengue SS. Nível de informação a respeito de medicamentos prescritos a pacientes ambulatoriais de hospital universitário. Cad Saude Publica 2000; 16(2):449-455.

36. Boonstra E, Lindbaek M, Ngome E, Tshukudu K, Fugelli P. Labelling and patient knowledge of dispensed drugs as quality indicators in primary care in Botswana. Qual Saf Health Care 2003; 12(3):168-175.

37. Marks JR, Schectman JM, Groninger H, Plews-Ogan ML. The association of health literacy and sociodemographic factors with medication knowledge. Patient Educ Couns 2010; 78(3):372-376.

38. Chau I, Korb-Savoldelli V, Trinquart L, Caruba T, Prognon P, Durieux P, Piketty C, Sabatier B. Knowledge of oral drug treatment in immunocompromised patients on hospital discharge. Swiss Med Wkly 2011; 141:w13204.

39. Moreira LB, Fernandes PF, Mota RS, Monte FS, Galvao RI, Sousa DF, Martins AM. Conhecimento sobre o tratamento farmacológico em pacientes com doença renal crônica. Rev Bras Cienc Farm 2008; 44(2):315-325. 
40. Moreira GC, Cipullo JP, Martin JF, Ciorlia LA, Godoy MR, Cesarino CB, Cordeiro JA, Lupino PL, Ciorlia G, Burdmann EA. Evaluation of the awareness, control and cost-effectiveness of hypertension treatment in a Brazilian city: populational study. J Hypertens 2009; 27(9):1900-1907.

41. Dias-da-Costa JS, Olinto MT, Bassani D, Marchionatti CR, de Bairros FS, de Oliveira ML, Hartmann M, Gabardo RM, Bennemann V, Pattussi, MP. Desigualdades na realização do exame clínico de mama em São Leopoldo, Rio Grande do Sul, Brasil. Cad Saude Publica 2007; 23(7):1603-1612.

42. Dias-da-Costa JS, Olinto MT, Gigante DP, Menezes AM, Macedo S, de Borba AT, da Motta GL, Fuch SC. Cobertura do exame citopatológico na cidade de Pelotas, Rio Grande do Sul, Brasil. Cad Saude Publica 2003; 19(1):191-197.

43. Fletcher SW, Fletcher RH, Thomas DC, Hamann C. Patients' understanding of prescribed drugs. J Community Health 1979; 4(3):183-189.

44. Arrais PS, Barreto ML, Coelho HL. Aspectos dos processos de prescrição e dispensação de medicamentos na percepção do paciente: estudo de base populacional em Fortaleza, Ceará, Brasil. Cad Saude Publica 2007; 23(4):927-937.

45. Santos V, Nitrini SM. Indicadores do uso de medicamentos prescritos e de assistência ao paciente de serviços de saúde. Rev Saude Publica 2004; 38(6):819826.

46. Woroniecki CL, McKercher PL, Flagler DG, Berchou R, Cook JA. Effect of pharmacist counseling on drug information recall. Am J Hosp Pharm 1982; 39(11):1907-1910.

Artigo apresentado em 19/07/2012

Aprovado em 20/10/2012

Versão final apresentada em 02/11/2012 